\title{
A Real-world Study on Prescription Pattern of Fosfomycin in Critical Care Patients
}

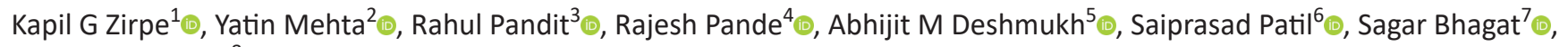
Hanmant Barkate ${ }^{8}$ (0)

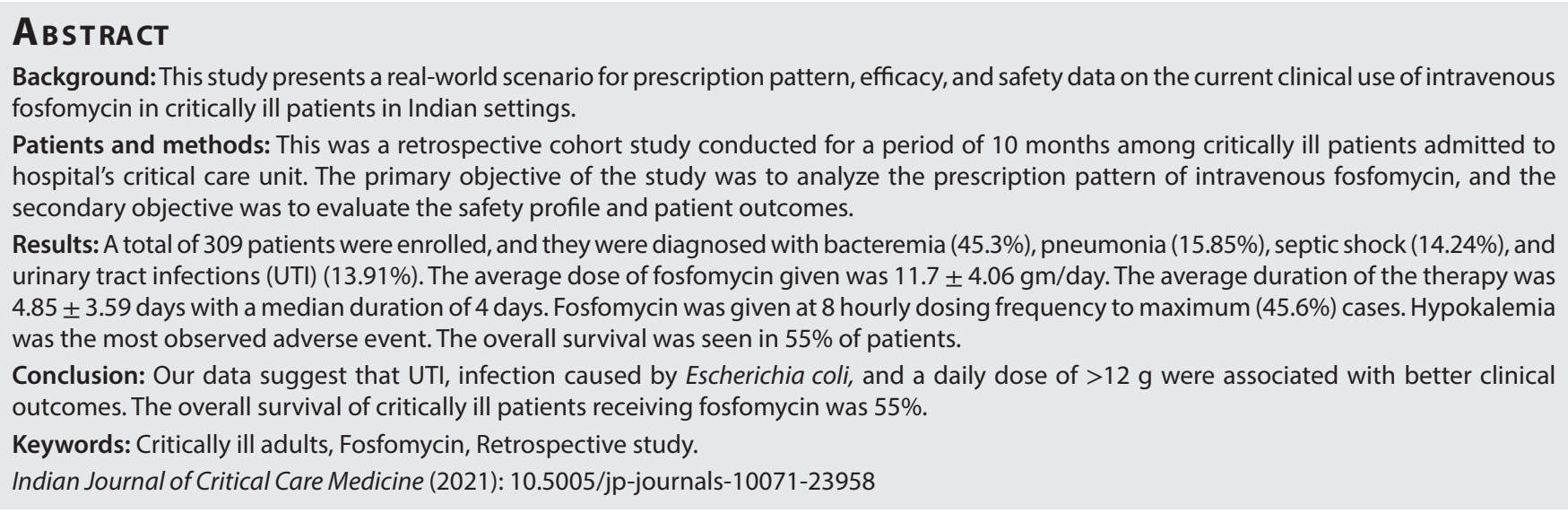

\section{INTRODUCTION}

Fosfomycin is a broad-spectrum, reemerging bactericidal antibiotic active against various gram-positive and gram-negative pathogens. ${ }^{1}$ Due to its unique chemical structure and the mechanism of action, fosfomycin does not exhibit cross-resistance with other antibiotics. ${ }^{2}$ Intravenous fosfomycin has been prescribed for various serious systemic infections, such as acute osteomyelitis, nosocomial lower respiratory tract infections, complicated urinary tract infections (UTI), bacterial meningitis, and bacteremia, in various countries. ${ }^{3,4}$ Standardization of fosfomycin dose for serious infections is needed, as in cases of severe infections, comparatively higher doses may be needed for the prevention of heteroresistant mutant selection. The daily dose used in clinical practice is anywhere between 12 and $24 \mathrm{~g}$ as two to four divided doses. ${ }^{5}$ A prospective, randomized, controlled clinical trial with fosfomycin in mono- and combination therapy, though having been initiated recently, is still ongoing.

Currently, data on the prescription pattern and safety of intravenous fosfomycin in the daily clinical practice, specifically in critically ill patients, with high bacterial load are still limited. Herein, we present a real-world scenario for prescription pattern and safety data on the current clinical use of intravenous fosfomycin (fosfomycin disodium inj) in critically ill patients in Indian clinical settings.

\section{Materials and Methods}

\section{Study Objective}

The primary objective of the study was to analyze the prescription pattern of intravenous fosfomycin in critically ill patients having positive bacterial cultures. The secondary objective was to evaluate the safety profile of fosfomycin and patient outcomes by means of assessing adverse events and all-cause mortality till day 30 , respectively.
${ }^{1}$ Department of Neuro Trauma Unit, Grant Medical Foundation, Pune, Maharashtra, India

${ }^{2}$ Institute of Critical Care and Anesthesia, Medanta-The Medicity, Gurugram, Haryana, India

${ }^{3}$ Department of Intensive Care, Fortis, Mumbai, Maharashtra, India

${ }^{4}$ Department of Critical Care Medicine, BLK Super Speciality Hospital, Delhi, India

${ }^{5}$ Department of Neuro Trauma Stroke Unit, Grant Medical Foundation's Ruby Hall Clinic, Pune, Maharashtra, India

6,8 Department of Global Medical Affairs, Glenmark Pharmaceutical Ltd, Mumbai, Maharashtra, India

${ }^{7}$ Glenmark Pharmaceutical Ltd, Mumbai, Maharashtra, India

Corresponding Author: Sagar Bhagat, Glenmark Pharmaceutical Ltd, Mumbai, Maharashtra, India, Phone: +91 9930553638, e-mail: sagar. bhagat@glenmarkpharma.com

How to cite this article: Zirpe KG, Mehta $Y$, Pandit R, Pande R, Deshmukh AM, Patil S, et al. A Real-world Study on Prescription Pattern of Fosfomycin in Critical Care Patients. Indian J Crit Care Med 2021;25(9):1055-1058.

Source of support: Nil

Conflict of interest: None

() Jaypee Brothers Medical Publishers. 2021 Open Access This article is distributed under the terms of the Creative Commons Attribution 4.0 International License (https://creativecommons.org/licenses/by-nc/4.0/), which permits unrestricted use, distribution, and non-commercial reproduction in any medium, provided you give appropriate credit to the original author(s) and the source, provide a link to the Creative Commons license, and indicate if changes were made. The Creative Commons Public Domain Dedication waiver (http://creativecommons.org/publicdomain/zero/1.0/) applies to the data made available in this article, unless otherwise stated.

\section{Study Design and Recruitment}

A retrospective cohort study was conducted for period of 10 months (March to December) among critically ill patients admitted to hospital critical care unit suffering from various serious infections and receiving at least 24 hours therapy of fosfomycin disodium injection. Patient's medical records were sought at each site by review of microbiology reports and bacteremia databases. Patients 
who had missing key data and death sooner than 24 hours after the index date were excluded from the study. Patients undergoing therapy with an active antibiotic for at least 2 days when blood cultures (BCs) were taken and having subsequent episodes of infection were also excluded.

Key data tracked for enrolled patients included demographics, medical, and surgical history, prior and concomitant medications, physical examination, weight, vital signs, Charlson comorbidity index, Pitts score, BC and aspartate aminotransferase test report, serum electrolytes, hematology, serum chemistry, urinalysis, assessment of adverse events or serious adverse events, and fosfomycin sodium inj. administration details.

\section{Study Endpoints}

Primary outcomes included analysis of indication, dose, frequency, and duration of fosfomycin among critically ill patients. Secondary outcomes included analysis of frequencies of adverse events in patients dosed fosfomycin. Further outcomes also comprise comparison of disease severity based upon various scores between survivors and nonsurvivors who were given fosfomycin.

\section{Safety}

Tracking of serum electrolytes, serum creatinine, and liver function tests for the 30-days' time period or until discharge/death (whichever occurred first) from patients' medical records was done to capture any significant finding. In cases of fosfomycin discontinuation due to adverse events, the timing and reason were recorded. Renal toxicity was defined as at least a doubling of the baseline creatinine value or a glomerular filtration rate decrease by at least $50 \%$, whereas severe hypokalemia was arbitrarily defined as any serum level $<3.0 \mathrm{mEq} / \mathrm{L}$.

\section{Results}

A total of 309 patients' dataset was found valid to be considered for the full analysis set (FAS). The average age of the population in FAS was $60.59 \pm 15.90$ years. The cohort consisted of 193 (62.45\%) males and 116 (37.54\%) females (Table 1).

Bacteremia with $45.3 \%$ of incidence was the most common diagnosis. The second most common diagnosis was pneumonia (15.85\%) followed by septic shock (14.24\%) and UTI (13.91\%). Cases diagnosed to be UTI had a significantly lower mortality proportion (11 out of 53) $(p<0.01)$ (Table 1). Other cases were skin and soft tissue infections (SSTI) (3.24\%) and infective endocarditis, osteomyelitis, meningitis, gynecological infection, and suspected carbapenem-resistant Enterobacteriaceae (CRE) infection $(<2 \%$ each). The proportion of the various diagnoses among survivors and nonsurvivors was not significantly different except in cases of UTI.

Immunocompromised patients consisted of $21.6 \%$ of the mortality group, which was significantly higher than survivors of $12.9 \%(p=0.042)$.

Among the organisms isolated from various culture sources collected from the cohort of critically ill patients, Klebsiella pneumoniae with 149 cases $(48.22 \%)$ was the most common bacterial isolate. With 49 cases (15.9\%) of occurrence, Escherichia coli emerged as the second most cultured bacteria with significantly lower mortality ( $21.76 \%$ survivor vs $8.63 \%$ nonsurvivors) $(p=0.001$ ). No association was observed between the isolated organism and the survival status of the patients.

The average duration of the fosfomycin therapy was $4.85 \pm$ 3.59 days with a median duration of 4 days. Fosfomycin was given at 8 hourly dosing frequency to maximum (45.6\%) cases. It was given
Table 1: Baseline characteristics of enrolled patients

\begin{tabular}{|c|c|c|}
\hline Overall average age (years) & \multicolumn{2}{|c|}{$60.59 \pm 15.90$} \\
\hline Male:Female & \multicolumn{2}{|c|}{ 193:116 } \\
\hline & \multicolumn{2}{|c|}{ Diagnosis } \\
\hline Diagnosis & No. of patients & Percentage \\
\hline Bacteremia & 140 & 45.30 \\
\hline Septic shock & 44 & 14.24 \\
\hline Pneumonia & 49 & 15.85 \\
\hline$|A|$ & 3 & 0.97 \\
\hline Infective endocarditis & 4 & 1.29 \\
\hline Osteomyelitis & 2 & 0.65 \\
\hline SSTI & 10 & 3.24 \\
\hline UTI & 53 & 13.91 \\
\hline Meningitis & 2 & 0.65 \\
\hline Gynecological infection & 1 & 0.32 \\
\hline Suspected CRE infection & 1 & 0.32 \\
\hline \multicolumn{3}{|l|}{ Organism } \\
\hline Enterobacter aerogenes & 3 & 0.9 \\
\hline Escherichia coli & 49 & 15.85 \\
\hline Klebsiella pneumoniae & 149 & 48.22 \\
\hline Proteus mirabilis & 3 & 0.9 \\
\hline Pseudomonas aeruginosa & 27 & 8.73 \\
\hline Serratia marcescens & 2 & 0.64 \\
\hline Staphylococcus spp. & 12 & 3.88 \\
\hline Mixed & 47 & 15.21 \\
\hline No growth & 17 & 5.50 \\
\hline
\end{tabular}

at 6 hourly and 12 hourly frequencies to 20.1 and $13.3 \%$ of cases, respectively. Dosing frequency of more than 12 hours, 3 hours, and stat dose was practiced sparingly (Table 2).

The average dose of fosfomycin given was $11.7 \mathrm{~g} / \mathrm{day}$. The average frequency of fosfomycin prescription was $9.13 \pm 4.45$ hours (3-24 hours). It was noted that $27.4 \%$ of cases given a daily dose of $\geq 12$ gm showed clinical resolution as compared to only $4.6 \%$ of cases receiving an average daily dose of $<12 \mathrm{~g}(p<0.001)$.

Hypokalemia was the most observed adverse event with the occurrence seen in a total of $62.1 \%$ of cases. However, its incidence was not significantly different between survivors and nonsurvivors. Surprisingly, higher average daily doses, that is, $\geq 12 \mathrm{~g}$, were associated with a lower incidence of hypokalemia $(48.4 \%$ in higher vs $75 \%$ in lower average doses) $(p<0.001)$. Hypernatremia was observed in a total of $24.3 \%$ of cases. It was significantly higher among nonsurvivors (31.7\%) as compared to survivors $(18.2 \%)(p=0.007)$. Hypernatremia was also recorded in a greater incidence among patients given higher average daily doses of $\geq 12 \mathrm{~g}(28.7 \mathrm{vs} 19.7 \%)(p=0.06)$ (Table 2$)$. Overall, the survival rate was $55.01 \%$ among patients in our study, with the highest for UTI (79.24\%) followed by bacteremia (52.14\%), pneumonia (44.89\%), and septic shock (43.18\%). Survival was better with E. coli (75.51\%) as a pathogen compared to Klebsiella pneumoniae (49.66\%). The average dose was $11.88 \pm 3.83 \mathrm{~g} /$ day for $4.71 \pm 3.81$ days.

\section{Discussion}

This retrospective study with 309 patients conducted by our group is among very few studies focused on understanding the 
Table 2: Prescription pattern, outcomes, and safety of fosfomycin

\begin{tabular}{lcc}
\hline Parameters & Mean & SD \\
\hline Average dose of fosfomycin (gm) & 11.76 & 4.06 \\
Average duration of therapy (days) & 4.66 & 3.68 \\
\hline Parameters & No. of patients & Percentage \\
\hline Fosfomycin dosing (gm/day) & & \\
\hline 3 & 3 & 1 \\
4 & 4 & 1.3 \\
6 & 62 & 20.1 \\
8 & 141 & 45.6 \\
12 & 41 & 13.3 \\
24 & 18 & 5.8 \\
$>24$ & 40 & 12.9 \\
\hline Outcomes & & 16.2 \\
\hline Discharged with clinical cure & 50 & 45 \\
Died & 139 & 23 \\
\hline Adverse events & & 61.5 \\
\hline No adverse events & 71 & 24.3 \\
Hypokalemia & 190 & \\
Hypernatremia & 75 & \\
\hline
\end{tabular}

prescription pattern and the use of intravenous fosfomycin in a real-world scenario. The wider use of fosfomycin against various infections was observed in our study, which is in concordance with previous studies. ${ }^{5}$

In real-world practice, fosfomycin has been used not only against infections of various bacterial origins but also at different difficult-to-treat infections, such as those involving biofilm formation. ${ }^{6}$ This justifies the prescription of fosfomycin at a higher rate in Klebsiella pneumoniae infection observed in this study. ${ }^{7}$ The properties of fosfomycin, including its tiny size, good volume of distribution, and tissue penetration, explain the use of fosfomycin in our study cohort for bacteremia, septic shock, infective endocarditis, and meningitis. ${ }^{8}$

The highest percentage of patients in fosfomycin-treated cohort included bacteremia cases. This high percentage of bacteremia patients treated with fosfomycin corroborates with few earlier studies that have shown the use of fosfomycin in combination with other agents for the treatment of bacteremia infections. ${ }^{9}$ Noticeably, the percentage of survivors in E. coliinfected group was significantly higher than nonsurvivors, suggesting the potential activity of fosfomycin against $E$. coli, which is in line with a previous study that reported $100 \%$ susceptibility of fosfomycin to $E$. coli isolates. ${ }^{10}$ The average dose of fosfomycin given to the overall population was $11.76 \pm 4.06 \mathrm{~g} / \mathrm{day}$, and the average duration of the therapy was $4.66 \pm 3.68$ days. Fosfomycin was given at 8 hourly dosing frequency to maximum (45.6\%) cases. We compared the prescription pattern of fosfomycin in our study with various other studies and their outcomes in two contexts, viz. dose and duration. The dose in our study was comparable to a study carried out in two European countries where an average dose of $13.7 \mathrm{~g} /$ day was given. Various other studies have also reported the dose of fosfomycin between 12 and $24 \mathrm{~g} /$ day. ${ }^{11,12}$ We noted that $27.4 \%$ of cases receiving an average daily dose of $\geq 12 \mathrm{~g}$ recovered completely compared to only $4.6 \%$ of cases given an average daily dose of $<12 \mathrm{~g}(p<0.001)$. Experts recommend that the dose of fosfomycin could be optimized in case of systemic infections. ${ }^{13} \mathrm{~A}$ multicentric ZEUS study recruiting patients for UTI suggested $18 \mathrm{~g}$ daily dose of fosfomycin with $6 \mathrm{~g}$ fosfomycin every 8 hours. ${ }^{14}$ Based on the observations from various studies, it has been found that on an average in the case of patients with normal renal function, the dose of fosfomycin could be in the range of 12-16 $\mathrm{g}$ of total daily dose administered in two to three doses. ${ }^{15-17}$

The maximum number of patients received fosfomycin at 8 hourly frequency that was supported by an in vivo study of intravenous fosfomycin disodium showing that effective plasma concentration for susceptible pathogens could be achieved up to 8 hours after intravenous administration. ${ }^{18}$ Eight-hour interval has been observed in various other studies also, such as ZEUS study as discussed above.

After intravenous administration of fosfomycin, sodium overload and hypokalemia are the most common adverse events observed. Every gram of intravenous fosfomycin contains $0.32 \mathrm{~g}$ of sodium. It has been postulated that fosfomycin leads to increased urinary excretion of potassium in the distal part of the renal tubules. In a French study, hypokalemia was reported in 19 of the 76 subjects, that is, $26 \%$ of patients. ${ }^{19}$ The authors reported that while potassium was administered in all patients, hypokalemia was found only when fosfomycin was administered in 30-60-minute infusions, while it did not occur when fosfomycin was administered for 4 hours. Similar to the above-reported studies, we also found hypokalemia as the most observed adverse event with the occurrence seen in a total of $62.1 \%$ of cases. Incidentally, the higher average daily dose (>12 g) subgroup had a significantly lower incidence of hypokalemia that could not be explained. Hypokalemia is a common manifestation seen in intensive care unit patients, and medications used in the management of various critical conditions are commonly associated with it. ${ }^{20}$ Therefore, clinicians should be aware of disturbances in sodium and potassium homeostasis and initiate adequate measures early to avoid further complications.

\section{Conclusion}

Our results indicate that bacteremia and pneumonia were the most common indication for fosfomycin prescription. Klebsiella pneumoniae was the most common pathogen. UTI and infection caused by $E$. coli were found to be associated with better clinical outcomes.

The overall survival in critically ill patients receiving fosfomycin was $55 \%$. The daily dose of $>12 \mathrm{~g}$ was associated with better clinical outcomes. Hypokalemia was the most frequent side effect reported.

\section{Limitations and Future Aspects}

The retrospective nature of the study posed few challenges like the availability of limited data on culture and sensitivity and resistance pattern to fosfomycin and other combination antibiotics presenting the usage pattern as empirical and less specific. Nonavailability of microbial curve data limited the efficacy outcome measures.

Future studies with double arms in Indian settings can throw more light on the use of fosfomycin in critical infections as targeted monotherapy and in combination with other antibiotics.

\section{OrCID}

Kapil G Zirpe $\odot$ https://orcid.org/0000-0002-8140-727X

Yatin Mehta $\odot$ https://orcid.org/0000-0002-0888-4774

Rahul Pandit (1) https://orcid.org/0000-0002-5846-3708 
Rajesh Pande (1) https://orcid.org/0000-0002-0149-727X

Abhijit M Deshmukh (1) https://orcid.org/0000-0001-5602-291X

Saiprasad Patil @ https://orcid.org/0000-0002-4937-3188

Sagar Bhagat $\odot$ https://orcid.org/0000-0002-0488-9359

Hanmant Barkate 다 https://orcid.org/0000-0003-4346-9194

\section{References}

1. Falagas ME, Kastoris AC, Kapaskelis AM, Karageorgopoulos DE. Fosfomycin for the treatment of multidrug-resistant, including extended-spectrum $\beta$-lactamase producing, Enterobacteriaceae infections: a systematic review. Lancet Infect Dis 2010;10(1):43-50. DOI: 10.1016/S1473-3099(09)70325-1.

2. Silver LL. Fosfomycin: mechanism and resistance. Cold Spring Harb Perspect Med 2017;7(2):a025262. DOI: 10.1101/cshperspect.a025262. PMID: 28062557; PMCID: PMC5287057.

3. Saiprasad P, Krishnaprasad K. Exploring the hidden potential of fosfomycin for the fight against severe gram-negative infections. Indian J Med Microbiol 2016;34(4):416-420. DOI: 10.4103/02550857.195379.

4. Docobo-Pérez F, Drusano GL, Johnson A, Goodwin J, Whalley S, Ramos-Martín V, et al. Pharmacodynamics of fosfomycin: insights into clinical use for antimicrobial resistance. Antimicrob Agents Chemother 2015;59(9):5602-5610. DOI: 10.1128/AAC.00752-15. Available from: https://aac.asm.org/content/59/9/5602.

5. Falagas ME, Vouloumanou EK, Samonis G, Vardakasa KZ. Fosfomycin. Clin Microbiol Rev 2016;29(2):321-347. DOI: 10.1128/CMR.00068-15.

6. Drobnic L, Quiles M, Rodríguez A. A study of the levels of fosfomycin in the cerebrospinal fluid in adult meningitis. Chemotherapy 1977;23(1):180-188. DOI: 10.1159/000222045. Available from: https:// www.karger.com/Article/ FullText/222045.

7. Bandeira M, Carvalho PA, Duarte A, Jordao L. Exploring dangerous connections between Klebsiella pneumoniae biofilms and healthcareassociated infections. Pathogens 2014;3(3): 720-731. DOI: 10.3390/ pathogens3030720.

8. Schintler MV, Traunmuller F, Metzler J, Kreuzwirt G, Spendel S, Mauric O, et al. High fosfomycin concentrations in bone and peripheral soft tissue in diabetic patients presenting with bacterial foot infection. J Antimicrob Chemother 2009;64(3):574-578. DOI: 10.1093/jac/ dkp230. Available from: https://academic.oup.com/jac/article-lookup/ doi/10.1093/jac/dkp230.

9. Rosso-Fernandez C, Sojo-Dorado J, Barriga A, Lavin-Alconero L, Palacios Z, Lopez-Hernandez I, et al. Fosfomycin versus meropenem in bacteraemic urinary tract infections caused by extended-spectrum -lactamase-producing Escherichia coli (FOREST): study protocol for an investigator-driven randomised controlled trial. BMJ Open 2015;5(3):e007363-e007363. DOI: 10.1136/bmjopen-2014-007363.
10. Maraki S, Samonis G, Rafailidis PI, Vouloumanou EK, Mavromanolakis E, Falagas ME. Susceptibility of urinary tract bacteria to fosfomycin. Antimicrob Agents Chemother 2009;53(10):4508-4510. DOI: 10.1128/ AAC.00721-09.

11. Hashemian SM, Farhadi Z, Farhadi T. Fosfomycin: the characteristics, activity, and use in critical care. Ther Clin Risk Manag 2019;15:525-530. DOI: 10.2147/TCRM.S199119. Available from: https://www.dovepress. com/fosfomycin-the-characteristics-activity-and-use-in-criticalcare-peer-reviewed-article-TCRM.

12. Zhanel GG, Walkty AJ, Karlowsky JA. Fosfomycin: a first-line oral therapy for acute uncomplicated cystitis. Can J Infect Dis Med Microbiol 2016;2016:1-10. DOI: 10.1155/2016/2082693. Available from: http://www.hindawi.com/journals/cjidmm/2016/2082693/.

13. Karageorgopoulos DE, Wang R, Yu XH, Falagas ME. Fosfomycin: evaluation of the published evidence on the emergence of antimicrobial resistance in gram-negative pathogens. J Antimicrob Chemother 2012;67(2):255-268. DOI: 10.1093/jac/dkr466.

14. Kaye KS, Rice LB, Dane AL, Stus V, Sagan O, Fedosiuk E, et al. Fosfomycin for injection (ZTI-01) versus piperacillin-tazobactam for the treatment of complicated urinary tract infection including acute pyelonephritis: ZEUS, a phase $2 / 3$ randomized trial. Clin Infect Dis 2019;69(12):2045-2056. DOI: 10.1093/cid/ciz181.

15. Apisarnthanarak A, Mundy LM. Use of high-dose 4-hour infusion of doripenem, in combination with fosfomycin, for treatment of carbapenem-resistant Pseudomonas aeruginosa pneumonia. Clin Infect Dis 2010;51(11):1352-1354. DOI: 10.1086/657249.

16. Michalopoulos A, Virtzili S, Rafailidis P, Chalevelakis G, Damala M, Falagas ME. Intravenous fosfomycin for the treatment of nosocomial infections caused by carbapenem- resistant Klebsiella pneumoniae in critically ill patients: a prospective evaluation. Clin Microbiol Infect 2010;16(2):184-186. DOI: 10.1111/j.14690691.2009.02921.x.

17. Miŕo JM, Entenza JM, Del Río A, Velasco M, Castañeda X, De La Mària CG, et al. High-dose daptomycin plus fosfomycin is safe and effective in treating methicillin-susceptible and methicillin-resistant Staphylococcus aureus endocarditis. Antimicrob Agents Chemother 2012;56(8):4511-4515. DOI: 10.1128/AAC.06449-11.

18. Sumano $\mathrm{LH}, \mathrm{Ocampo} \mathrm{CL}$, Gutierrez $\mathrm{OL}$. Intravenous and intramuscular pharmacokinetics of a single-daily dose of disodium-fosfomycin in cattle, administered for 3 days. JVet Pharmacol Ther 2007;30(1):49-54. DOI: 10.1111/j.1365-2885.2007.00812.x.

19. Florent A, Chichmanian RM, Cua E, Pulcini C. Adverse events associated with intravenous fosfomycin. Int J Antimicrob Agents 2011;37(1):82-83. DOI: 10.1016/j.ijantimicag.2010.09.002.

20. Gennari FJ. Disorders of potassium homeostasis. Hypokalemia and hyperkalemia. Crit Care Clin 2002;18(2):273-288, vi. DOI: 10.1016/ s0749-0704(01)00009-4. 